\title{
A Framework for End-to-End Service Differentiation: Network Planes and Parallel Internets
}

\author{
M. Boucadair and P. Lévis, France Telecom \\ D. Griffin, University College London \\ N. Wang, M. Howarth, and G. Pavlou, University of Surrey \\ E. Mykoniati and P. Georgatsos, Algonet \\ B. Quoitin, Université Catholique de Louvain \\ J. Rodríguez Sánchez and M. L. García-Osma, Telefónica
}

\begin{abstract}
This article presents a technology-agnostic and a multi-dimensional (i.e., routing, forwarding, and traffic management dimensions) approach for the management of IP network resources to ensure service differentiation with both intra- and inter-domain scope. This article introduces the network plane (NP) and parallel Internets (PI) concepts for achieving service differentiation. Based on these concepts, a functional architecture together with a business model is presented. In addition, this article describes how the proposed approach can become a promising platform for the IP multimedia subsystem (IMS), with the objective of providing end-to-end QoS-enabled multimedia delivery across multiple providers to replace the flow-based reservation mode known as the VoIP resource reservation framework.
\end{abstract}

\section{General Context}

The emergence of new services such as video streaming and IP telephony requires IP networks to provide stringent guarantees - not only in terms of traditional quality of service (QoS) metrics - but also in terms of availability (e.g., five nines for telephony) and robustness during emergency situations. From the earliest stage of IP networking, proposals have aimed to capture and support the requirements of various services, especially in the realm of forwarding and routing. In 1994, the Nimrod initiative [1] was launched within the Internet Engineering Task Force (IETF) with the goal of providing service-specific routing in the presence of multiple constraints imposed by operators and end users. RFC1992, one of the key documents produced by the Nimrod initiative, states that inter-network connectiv- ity and services should be represented by maps at multiple levels of abstraction. Nevertheless, this recommendation was never implemented. Additionally, QoS forwarding mechanisms such as IntServ [2] and DiffServ [3] were proposed but were not widely introduced into operational networks. ${ }^{1}$ The exceptions are practices adopted by operators, such as enforcing shaping and policing rules and using marking techniques to distinguish flows only at the access segments of their IP networks. This is due to the complexity and the lack of clear views on the manageability of such mechanisms and also to the fact that operators are not ready to refrain from their practices related to over-provisioning in favor of sophisticated traffic engineering techniques.

\section{QOS HURDLES IN 3GPP ARCHITECTURES}

Today, voice over IP (VoIP) is one of the major fields of service innovation, and most service providers plan (if they have not yet started) to migrate their public switched telephone network (PSTN) infrastructures to IP. For this goal, IP multimedia subsystem (IMS) [4], telecoms, Internet converged services, and protocols for advanced network [5] (TISPAN) architectures have been specified by the 3rd Generation Partnership Project (3GPP) community to meet the requirements of service providers. ${ }^{2}$ As far as QoS requirements are concerned, 3GPP documentation introduces the notion of QoS class but does not clearly define this notion. TS 23.107 identifies four QoS classes: conversational class, streaming class, interactive class, and background best effort. But TS 22.105 makes use of four groups of applications in terms of $Q o S$ requirements and points out that there is no strict one-to-one mapping between these groups and the classes as defined in TS 23.107. However TS 22.105 uses exactly the same names for its taxon- 
omy as those of TS 23.107. The following key issue results: is a QoS class defined in terms of QoS parameter values, or is it defined in terms of QoS requirements for a group of applications?

In addition, $3 \mathrm{GPP}$ relies on DiffServ to provide network services with the requested QoS parameters. Unfortunately, the statement DiffServ is used to provide $Q o S$ says very little about how the network actually can be engineered to deliver the requested QoS. It is true that it has never been the intent of QoS standards to provide information on how the actual network engineering is to be done. However, a significant gap does exist between the way network objectives are presented in terms of numerical values for delay, jitter, and loss and the way DiffServ per hop behaviors (PHBs) are defined. Creating best current practices (BCP)-type documents could provide valuable information to try to narrow this gap. The engineering of QoS requirements, as well as robustness and availability requirements, do not appear to be addressed by $3 \mathrm{GPP}$, although the assumption is made that a QoS-enabled network is available and that QoS can be requested on a per application-flow basis, especially during the session establishment, which is expressed as a session description protocol (SDP) offer. The success of such a session is a necessary condition for the reservation of appropriate resources in both directions of the call. An example of implementing this mode is the QoS preconditions as defined in [6]. This mode has several drawbacks, such as increasing the connection set up and release times, especially when crossing multiple telephony domains. Moreover, 3GPP specifications do not detail how to check the validity of the QoS requirements enclosed in SDP offers, what the interface between the VoIP signaling protocols and the QoS enforcement mechanisms is, how to validate the required QoS in both call directions, how requested QoS will be guaranteed, or how to ensure coherency of multimedia treatment when crossing several autonomous systems and IP telephony domains.

\section{Article Structure}

To handle the aforementioned challenges, mainly QoS and robustness, the A liGhtweight approach for viable end-to-end IP-based QoS services (AGAVE) project introduces the concepts of network plane (NP) and parallel Internets (PI) [7, 8], a novel transport platform that offers end-to-end service differentiation across the Internet. The proposed approach does not require a single Internet-wide architecture or universal deployment. This article presents these concepts and associated functions. The article also describes how IMS can make use of the AGAVE platform to offer QoS-enabled multimedia services.

This article is structured as follows. We present the adopted business model. We define network plane and parallel Internet concepts and provide examples of techniques to implement them. We describe the AGAVE functional architecture. Finally, we highlight the invoked AGAVE functional blocks when deploying two scenarios of NP/PI realization.

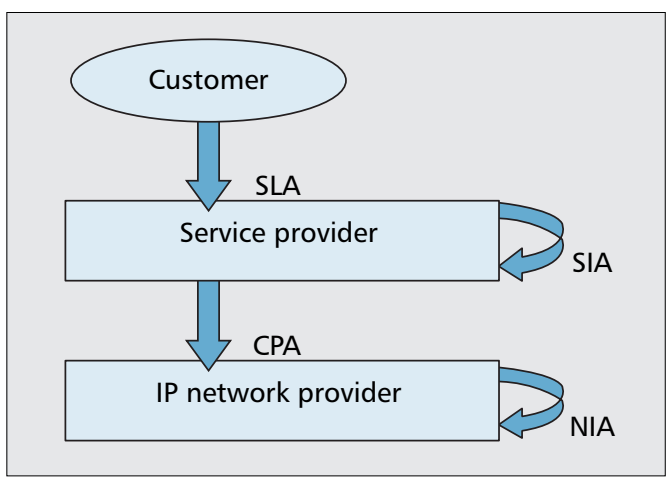

Figure 1. Business model.

\section{Reference Business Model}

The emergence of new players, such as Skype and Yahoo! in the telephony service market, as well as the trend for traditional telcos to migrate their services to run over all-IP networks, is indicative of the separation of service and network planes. This is leading to a distinction between the service provider (SP) and the IP network provider (INP) business roles (Fig. 1). It should be noted that business roles do not necessarily map one-to-one to distinct business entities; a business entity may implement more than one role.

INPs offer IP connectivity to SPs and do not offer their services directly to end customers. For expanding the scope of their IP connectivity, INPs interact with each other on a one-to-one relationship basis regulated by INP interconnection agreements (NIAs). An NIA specifies the QoS and availability performance of the traffic exchanged between the INPs, the scope and the profile of the traffic entitled to the agreed performance, and identifiers to capture distinct flows for providing differentiated treatment.

SPs offer IP-based services to end customers. SPs deploy the infrastructure required for the provisioning of the offered services, for example, VoIP gateways or IP video-servers. To fulfill the IP connectivity aspects of their services, SPs establish connectivity provisioning agreements (CPAs) with underlying INPs. Similarly to NIAs, CPAs specify the performance, constraints, and identifiers of the service traffic entering the INP network from the SP sites. Beyond the connectivity specified therein, the INP offers to the SP the means to control the connectivity provisioning, such as setting policing and routing rules and receiving feedback reports. The specific provisioning rules and required feedback also are agreed upon during the CPA negotiation.

To expand the scope of offered services, SPs interact with each other on the basis of SP interconnection agreements (SIAs). The content of an SIA is service-specific, for example, a VoIP SIA may include telephony performance metrics, such as average success rate or simultaneous calls capacity.

Customers are the target recipients of the services offered by an SP. Services are offered on the basis of service level agreements (SLAs), capturing the terms and conditions for the provision and use of the services.

\section{The emergence of new players, such as Skype and Yahoo! in the telephony service market, as well as the trend for traditional telcos to migrate their services to run over all-IP networks, is indicative of the separation of service and network planes.}




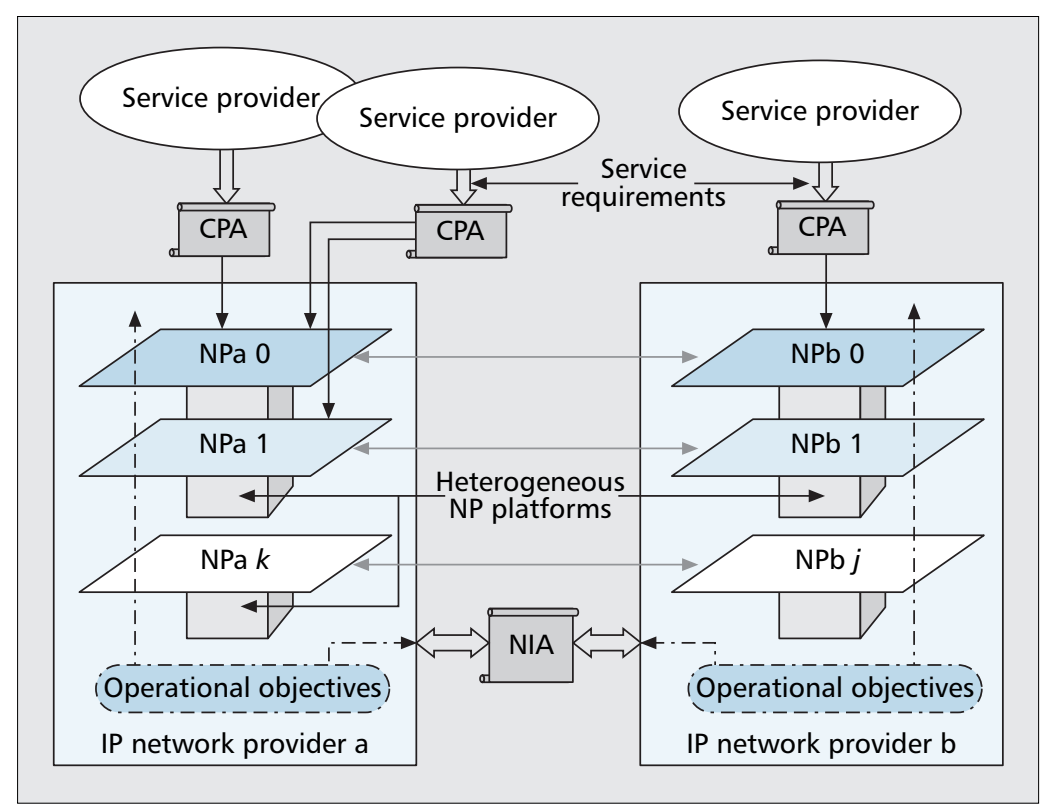

Figure 2. NP and PI realization.

\section{Network Plane and Parallel InTERnets Concepts}

AGAVE introduces network planes to differentiate the delivery behaviors experienced by IP flows when crossing an IP realm managed by a single INP. The NP notion is internal to INPs, and its engineering can be undertaken before or after the formulation of service requirements as expressed by SPs. In addition to traditional QoS metrics, such as delay and packet loss, requirements such as availability also are considered. It is up to the INP to plan/select/(re-)engineer its NPs to meet these SP requirements. A given NP can be used to convey service traffic managed by the same or distinct SPs in an aggregate fashion. To fulfill the service requirements specified in the CPA, INPs must engineer corresponding NPs within their own network. Technically, an NP can be engineered through the combined tuning of several processes, which span one or more of the following dimensions:

- Routing dimension: To support heterogeneous service requirements, different paths can be implemented for individual NPs. Routing differentiation can be implemented at several levels, for example:

-Assigning dedicated topologies to maintain several routing adjacencies towards the destination.

-Assigning dedicated path selection configurations so that multiple path selection configurations (e.g., routing metrics) can be installed, each dedicated to one specific NP. ${ }^{3}$

-Configuring dedicated fast reroute procedures for service resilience purposes, such as pre-configuring backup paths/topologies inside high availability NPs.

diverse paths can be simultaneously maintained between individual ingress/egress pairs to support different service requirements from individual NPs.

level, an INP can engineer its IP forwarding mechanisms so as to provide different packet scheduling behaviors by configuring different policies in a common scheduler, assigning dedicated scheduling resources, differentiating dropping policies, differentiating failure detection means, and so on.

- Resource management dimension: The treatment experienced by IP packets can be differentiated by different shaping and policing, as well as the degree of traffic multiplexing, also denoted as the overprovisioning factor.

INPs can select the most appropriate combination of mechanisms to implement specific NPs according to the service requirements. Furthermore, an INP takes into account its own operational objectives such as manageability, scalability, and resource optimization to provide cost-efficient NP realization. In the forwarding dimension, DiffServ is a common platform for supporting service differentiation. As far as the routing dimension is concerned, multi-topology routing mechanisms [9-12] are regarded as suitable platforms for supporting service differentiation both within and across NPs. Specifically, dedicated routing configuration, such as multitopology-open shortest path first (OSPF) link weight setting, multi-protocol-border gateway protocol (BGP), and QoS-enhanced BGP tweaking, can be performed on top of different routing topologies, each serving a specific NP. Additionally, other mechanisms can be applied for implementing NPs: by using the functionality of explicit routing and resource reservation of resource reservation protocol-traffic extension (RSVP-TE), dedicated label switched paths can be constructed to support hard QoS guarantees. Alternatively, QoS overlay routing and IP tunneling [13] techniques can be used for realizing NPs, with less stringent requirements such as better-than-best-effort services. As far as service resilience is concerned, IP/multiprotocol label switching (MPLS) fast rerouting techniques [14] can be used. A general overview of NP realization is described in Fig. 2.

The concept of parallel Internets is introduced as an innovative way to enable end-to-end service differentiation across multiple INPs. Specifically, PIs are constructed through horizontal interconnection of NPs across individual INPs. In doing so, INPs must negotiate and establish NIAs between each other to bind NPs with similar service characteristics and apply specific mechanisms in the control/data plane to enforce the realization of individual PIs. Each can have a dedicated inter-domain topology, routing policy, and forwarding behavior, and so on. A salient novelty of the proposed approach is that each instance of PI is not necessarily implemented with a homogeneous platform across multiple INPs. This aspect provides high flexibility for cooperating INPs to make local decisions in binding their own NPs to the PI. Later, two examples will be illustrated to show how PIs can be realized in practice.

\section{AGAVE Functional ArChitecture}

\section{OVERVIEW}

This section analyzes the interactions between the business roles of customer, SP, and INP and describes the functional blocks required to support these interactions, focusing in particular on 


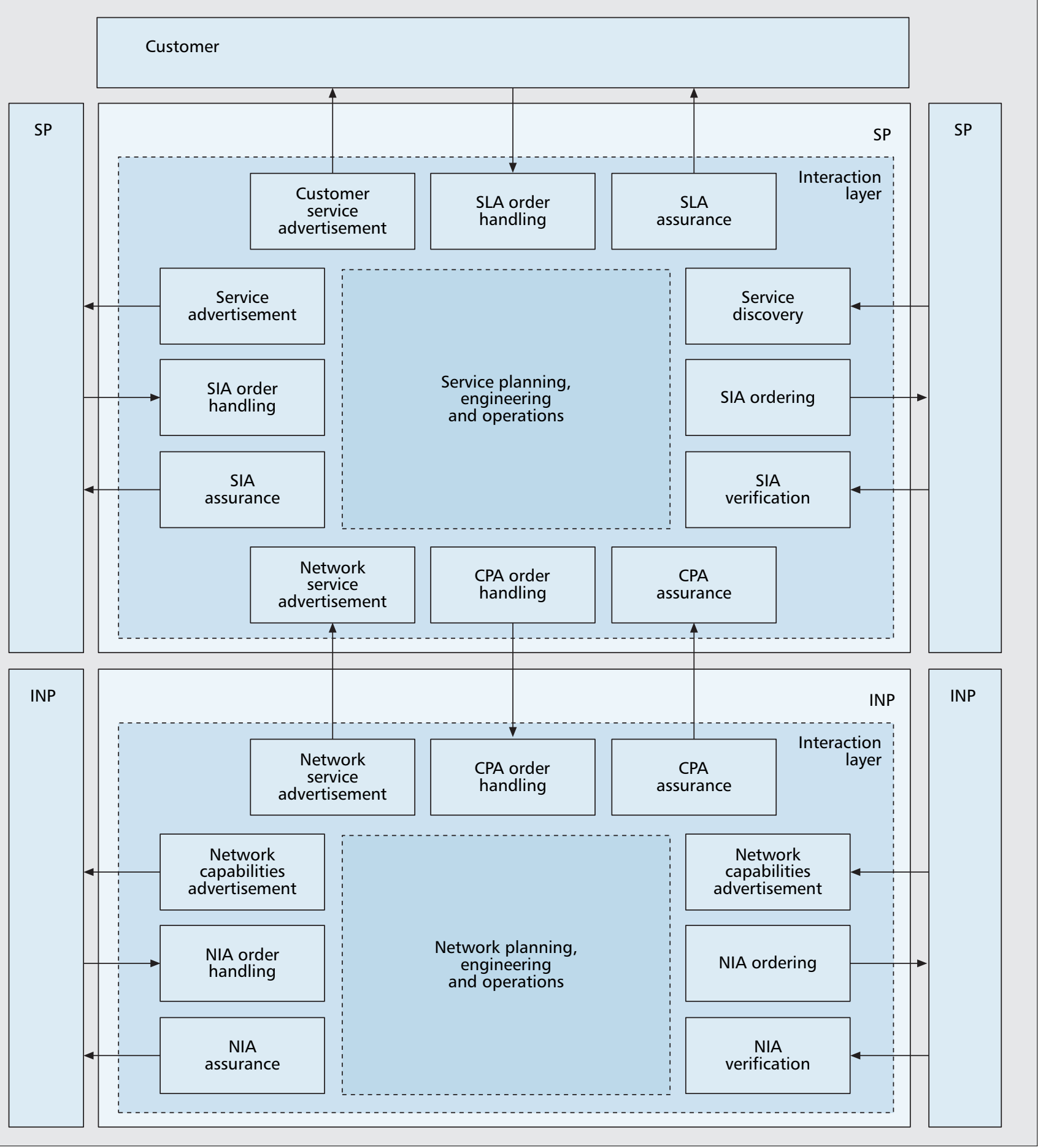

Figure 3. AGAVE functional architecture: interactions viewpoint.

the internal functionality required to plan, engineer, and operate network planes and parallel Internets within an INP.

Building on the business model discussed previously and depicted in Fig. 1, each agreement - CPA, NIA, SIA, and SLA - is supported by three sets of functional entities corresponding to the three phases of the contractual relationships (Fig. 3). Each set is comprised of a pair of corresponding functional blocks in the business entities operating in the customer and provider roles pertinent to each agreement. Service advertise- ment and discovery blocks conduct pre-agreement interactions; agreements are subsequently negotiated via ordering and order-handling blocks; and post-agreement, the performance of the service is monitored by verification and assurance functions.

This interactions-focused view hides the complexity of internal SP and INP functional blocks contained in the service/network planning, engineering, and operations meta-blocks. The functional blocks of the INP are further decomposed as follows. 


Three different
perspectives of the
INP operational
activities are
identified. The first is
the commercial
view; the second is
concerned with
network-wide
optimization of the
INP resources;
the third focuses on
network engineering
and the implementa-
tion and configura-
tion details of the
NPs/Pls.

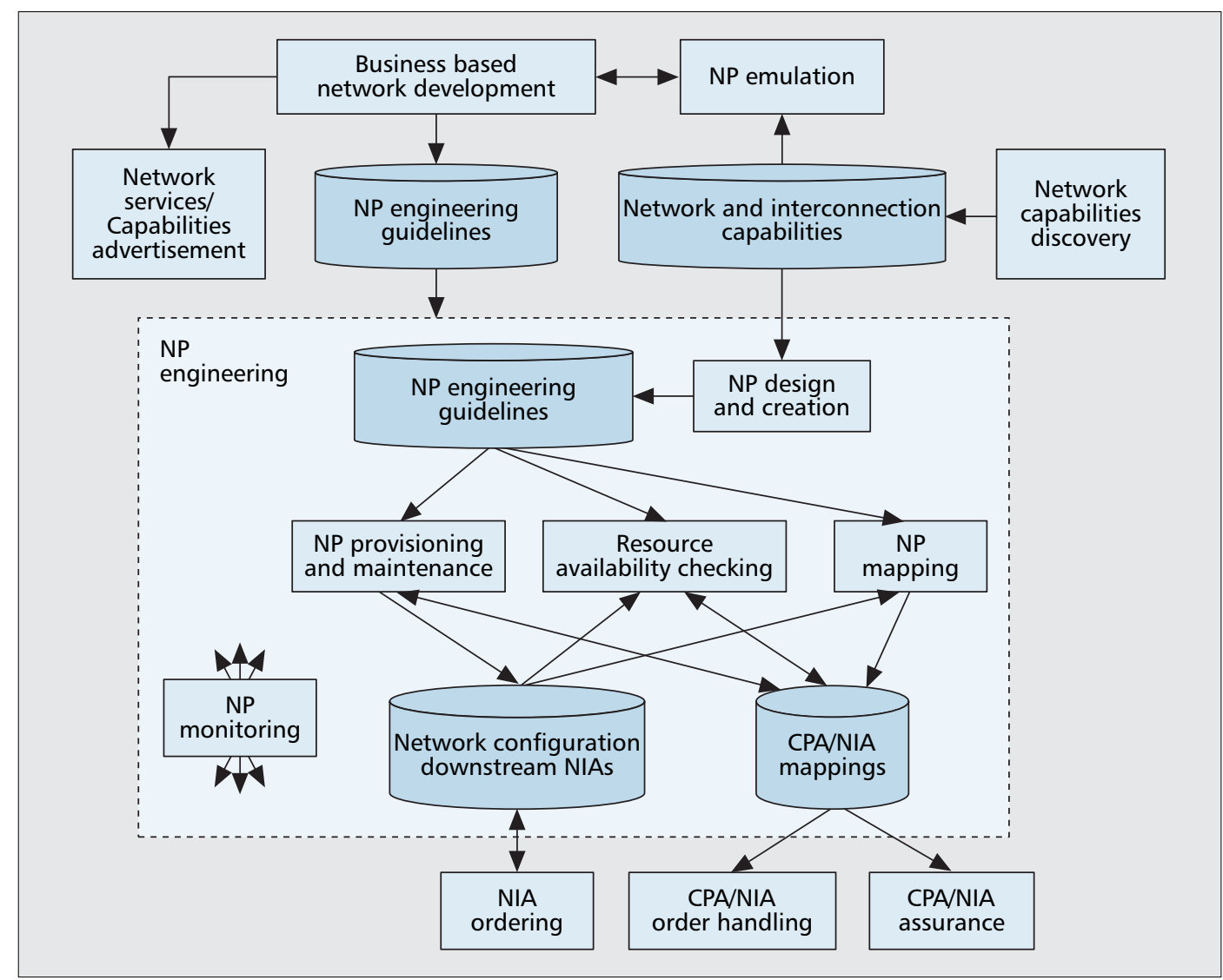

Figure 4. INP functional decomposition.

\section{RATIONALE}

The rationale behind the decomposition of functionality of the INP is to build a business-process view of the planning, management, and operations tasks of the network. The goal is to mirror the internal organizational structure of a typical INP, the steps involved in building NPs and PIs to support CPAs and NIAs with SPs and customer INPs, and to model the interactions between the functional entities.

Three different perspectives of the INP operational activities are identified. The first is the commercial view, which focuses on defining and ultimately selling connectivity services to SPs and customer INPs. Its main concern is to maximize the profit of the INP. The second perspective is concerned with network-wide optimization of the INP resources; given the services to be accommodated, their QoS and availability requirements, and the anticipated demand. This is where NPs and PIs and their overall realization objectives are defined. The third perspective is the one that focuses on network engineering and the implementation and configuration details of the NPs/PIs. The later view is heavily dependent on the technological aspects of the mechanisms selected for NP realization.

Although all three business processes are concerned with INP management and operations, they each have different perspectives and concerns as described previously, and they must communicate with one another to achieve the network-level configurations that ultimately sup- port the business objectives of the INP. The functional architecture facilitates this by defining clear boundaries between the entities implementing the decision-making processes and specifying the interactions between them, based on issues of common concern. The interactions should be based on information models at an appropriate level of abstraction but with sufficient detail to enable the delegation of tasks from the higher to lower levels and the reporting of state and problems encountered in the reverse direction. The specification of the information entities is outside the scope of this article but can be found in [8].

Figure 4 shows the functional blocks of the INP. The commercial perspective is handled primarily by the business-based network development block, supported by NP emulation and network capabilities discovery/advertisement. Network-wide optimization concerns are dealt with by $N P$ design and creation, while the detailed network engineering and configuration tasks are located in NP provisioning and maintenance. The functional blocks are described in more detail in the following paragraphs, and their interactions are illustrated through two scenarios in the following section.

\section{Functional Blocks Description}

Business-based network development sets the targets for the NP engineering components to fulfill, specifically, the network services to be supported and the guidelines for handling the demand for these services. Target network services are expressed in terms of QoS and availability per- 
formance metrics and are optionally restricted to a defined set of local or remote destinations.

NP emulation provides the business-based network development with data to support its decision-making process regarding the impact (financial, engineering, service capabilities, etc.) of accepting new connectivity requests, introducing new connectivity capabilities, enhancing the infrastructure, establishing new interconnections, and so on. A key purpose of NP emulation is to allow business-based network development to make deterministic decisions on the introduction of new services, increasing/reducing the traffic load of existing services, and other what-if scenarios by examining the impact of these changes on network performance and ultimately profitability, without the requirement to be aware of the technical details of how the services are engineered/deployed.

The NP design and creation functional block is responsible for the off-line dimensioning of network planes prior to their actual configuration into the operational network of an INP. The design and creation phase produces high level specifications of the network planes in terms of qualitative and quantitative parameters associated with routing, forwarding, and resource management dimensions. Therefore, the role of NP design and creation includes the definition of NPs in terms of their targeted QoS and availability capabilities and realization guidelines specific to the employed technologies to fulfill the business-based network development targets.

NP provisioning and maintenance undertakes the actual realization of network planes and parallel Internets and produces the appropriate network configuration, based on the technologies selected and the realization guidelines provided by the NP design and creation. It also produces the appropriate concrete network configuration data and NIA orders to be negotiated and established with neighboring INPs through the services of the NIA ordering block. This information is stored in the network configuration and downstream NIAs database in Fig. 4.

$N P$ mapping produces candidate CPA/NIA mappings to NPs and PIs on the basis of compatibility of the CPA/NIA requirements to the capabilities of the NPs and PIs. The produced CPA/NIA mappings are used by resource availability checking to deduce the admission or rejection of incoming CPA/NIA requests by comparing the capacity in the engineered NPs with the demand of the CPA/NIAs. NP provisioning and maintenance also uses the CPA/NIA mappings to actually accommodate the CPA/NIA traffic demand. The mappings are stored within the CPA/NIA mappings database in Fig. 4.

Data gathered by NP monitoring is used to generate notifications and reports for the $C P A / N I A$ order handling and CPA/NIA assurance to forward to SPs and upstream INPs, for the online traffic engineering functions in NP provisioning and maintenance, for resource availability checking to derive appropriate multiplexing factors, and for the NP design and creation and NP emulation and business-based network development functions to formulate a high level view of the network performance.

\section{AGAVE FUNCTIONAL ARCHITECTURE AT WORK}

This section illustrates the invoked functional blocks and associated interactions to implement NPs and PIs, employing different routing and forwarding techniques. The first scenario QoS-inferred parallel Internets is an ideal solution for a community of adjacent INPs who want to collaborate with one another to offer end-toend QoS across their networks. The second scenario - better-than-best-effort service, aims to provide less strict QoS between non-adjacent domains exchanging high traffic volume.

\section{QOS-INFERREd PARALLEL INTERnetS}

The following QoS-inferred parallel Internet scenario relies on the use of DiffServ, the metaQoS-class [15] concept, and the QoS-enhanced BGP protocol [12]:

- The meta-QoS-class concept assumes a universal understanding of QoS-sensitive application requirements. Because wherever customers are connected, they experience the same QoS difficulties and are likely to express similar QoS requirements to their respective providers, these providers are likely to operate similar NPs, each particularly designed to support services with the same constraints.

- QoS-enhanced BGP is an enriched version of the BGP protocol proposed to support QoS requirements for the deployment of QoS-based services across several domains. Two modes of activation of QoS-enhanced BGP to extend the network planes beyond the boundaries of an INP may be envisaged: either to configure several QoSenhanced BGP sessions, each dedicated to a given NP, or to activate a single QoSenhanced BGP session that will multiplex reachability information of all involved NPs.

Within this scenario, each INP domain is engineered to support a limited number of per domain behaviors (PDBs) through NP engineering functions (i.e., NPs are implemented as PDBs), for example, one PDB to convey conversational traffic and one for best-effort traffic. The dimensioning of these PDBs, including individual $\mathrm{PHB}$ profiles and associated differentiated services code point (DSCP) values are defined by NP design and creation and enforced within networks nodes by NP provisioning and maintenance functions. These PDBs are classified to well-known meta-QoS-classes by the NP design and creation. Each INP advertises, through its network-capabilities advertisement function, the meta-QoS-classes it supports. Other INPs can discover these capabilities through network-capabilities discovery and therefore request NIAs with the advertising INP to make use of the offered meta-QoS-class via the invocation of an NIA ordering/NIA order handling interface. When NIAs agree (results of NIA ordering and NIA order handling), each peering INP activates QoS-enhanced BGP per meta-QoS-class through NP provisioning and maintenance functions. The resulting QoS-
NP provisioning and

maintenance

undertakes the

actual realization of

network planes and

parallel Internets and

produces the

appropriate network

configuration, based

on the technologies

selected and the

realization guidelines provided by the NP design and creation. 
To achieve the

company's

objectives, two

different network

planes are used in

each INP where the

site is located: NP1,

dedicated to low

latency service that

accounts for a small

proportion over the

overall traffic and

NP2, used for

best-effort traffic.

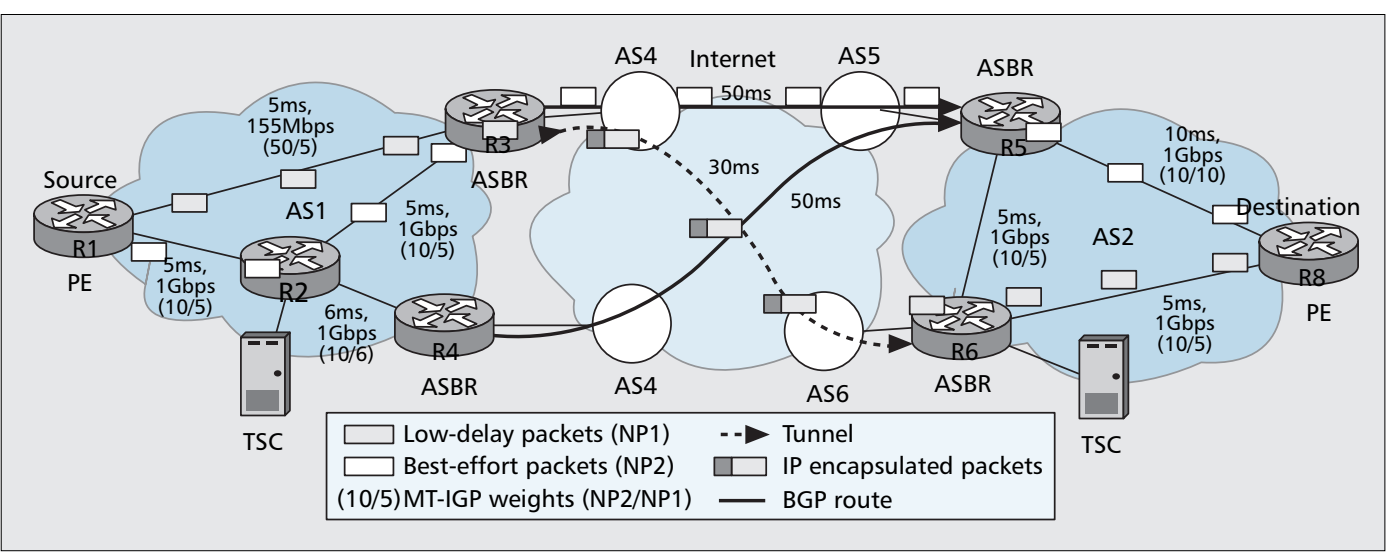

Figure 5. Better-than-best-effort service between two cooperating INPs.

enabled Internet can be viewed as a set of PIs, each offering QoS service levels associated with a specific meta-QoS-class and running distinct instances of QoS-enhanced BGP. The NIA agreement for a meta-QoS-class makes it possible for INPs to benefit from their neighbor's inter-domain QoS capabilities and enables them to reach anywhere in the QoS-Internet for that specific meta-QoS-class. The NP mapping function assigns identifiers (one for incoming traffic and another one for outgoing traffic) to be used in the inter-domain links. These identifiers enable the identification of the local PDB to be used to treat the traffic and therefore, the PI in which flows will be routed.

\section{Better-Than-Best-Effort Service}

In this second scenario, we combine multi-topology routing and IP tunneling techniques to improve inter-domain forwarding performance. We illustrate this combination based on the following example (Fig. 5). A company uses VoIP to place calls between its sites that are geographically spread in multiple domains owned by different INPs (AS1 and AS2). Its objectives are to minimize the end-to-end delay between its sites and simultaneously to balance the traffic load at each site.

To achieve the company's objectives, two different network planes are used in each INP where the site is located: NP1, dedicated to low latency service that accounts for a small proportion over the overall traffic and NP2, used for best-effort traffic. It is the role of NP design and creation to define the NPs and specify how each NP is implemented. In this example, the MultiTopology Internet Gateway Protocol (MT-IGP) supports two sets of link weights, one that is optimized for providing the lowest latency intradomain paths and the other that is designed to balance the overall traffic load within the domain. NP mapping is responsible for assigning traffic flows to a specific NP according to the constraints handled by the CPA order handling. Traffic flows between Session Initiation Protocol (SIP) proxy servers and between VoIP customers, and their outbound/inbound SIP proxy servers are attached to NP1; other traffic flows are attached to NP2. The assignment of traffic flows onto NPs can be based on packet fields (DSCP or source/destination ports). Based on these assignments, we know the part of the traffic matrix that is supported by each NP.

Given the fact that individual INPs containing the company sites are not necessarily adjacent to each other, it is not possible to achieve strict end-to-end delay between these sites only by the two ends of the INP along the entire inter-domain path without interfering with intermediate domains. As to what concerns the binding between the NPs in different INPs containing these sites, it is the responsibility of the centralized tunneling service controller (TSC) installed inside each INP to discover and select the interdomain paths with the best monitored one-way delay between the local and remote sites for the traffic assigned to NP1. The TSC service can be run on a separate workstation or as part of a router. TSC implements the following functional blocks: network capabilities discovery and advertisement, NP monitoring, and NP provisioning and maintenance. The operation of the TSC is as follows. First, the TSC discovers the possible ingress routers of the remote sites due to network capabilities discovery and advertisement. A communication is established with each remote site to discover its ingresses. Based on an exploration of the $\mathrm{BGP}$ routes received by the local AS border routers, the TSC identifies the egress routers that can reach the ingresses in the other sites. Then, the TSC performs a measurement of the latency between each pair of local egress and remote ingress requests (NP monitoring). Consequently, it selects the lowest delay paths. Final$1 y$, the inter-site paths are configured in the network, using the NP provisioning and maintenance block. If tunnels are required, NIAs are established with the corresponding remote sites that result in tunnel establishment. Additionally, local routers must be configured to forward NP1 traffic destined to remote sites through the selected paths. In the example of Fig. 5, the default routes selected by BGP to reach AS2 from AS1 have the longer delays (50 ms). A tunnel can be used to reach AS2 through a lower delay path (30 ms), exiting AS1 at R3 and entering AS2 at R6.

The off-line traffic engineering (TE) engine in NP provisioning and maintenance selects the IGP link weights that must be configured in the routing topology supporting each NP. The objective of the TE engine is to minimize the delay 


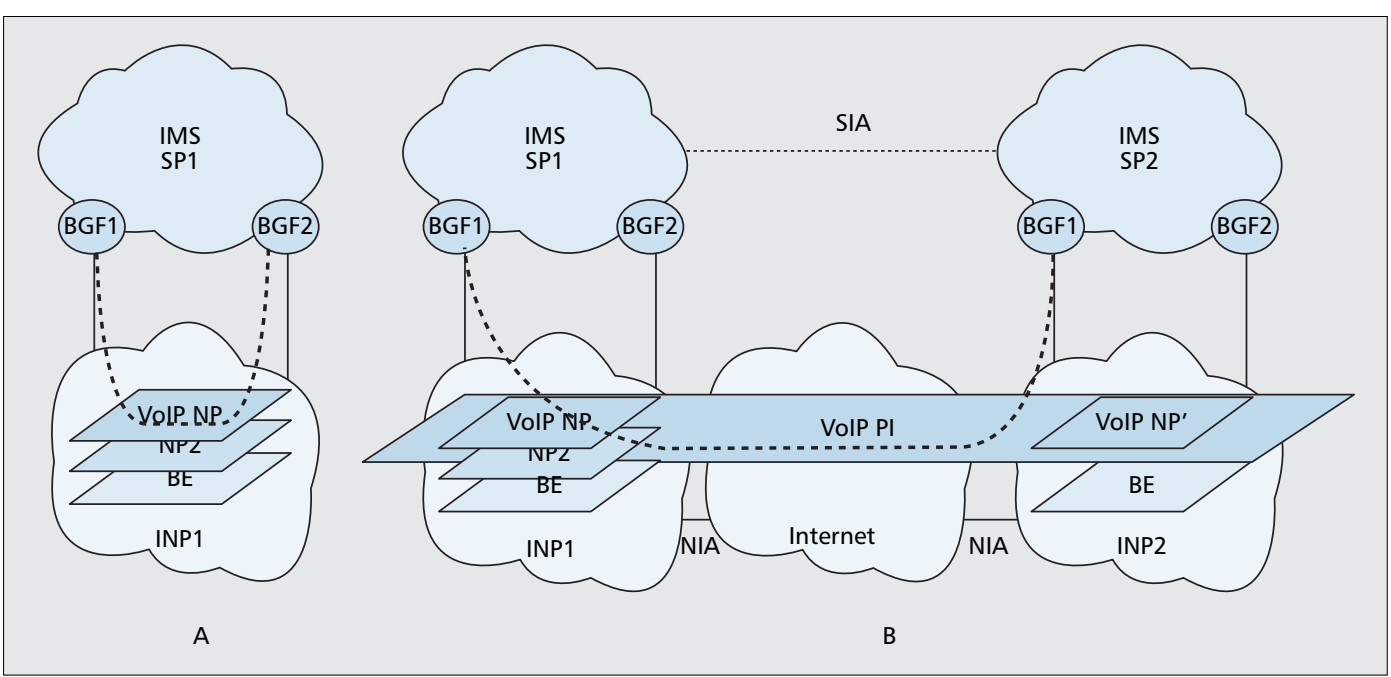

Figure 6. IMS, NPs, and PIs.

along the paths in NP1 while balancing the overall traffic load of NP1 and NP2 on the network resources. A typical realization of this optimization is to assign link weights to the routing topology of NP1 so as to select minimum delay paths and to assign link weights to the topology supporting NP2 to spread the traffic load within the network.

\section{IMS INTERACTION WITH AGAVE}

AGAVE offers an open interface for an SP to express its service requirements including QoS and availability. Owing to this interface, an SP need not be aware of the IP engineering operations executed by the underlying INP. The IMS-based SP can indicate its service requirements through CPAs, and there is no longer a need to define the bearer classes 4 for IP clouds. Underlying INPs run their NP design and creation machinery to meet these requirements. The process is transparent for an IMS-based $\mathrm{SP}$, decoupling VoIP signaling from the techniques that ensure QoS at the IP level. With this approach, IP resources are not reserved per call but per call aggregates and the IMS functions, for example, policy decision function (PDF) or resource admission control function (RACF), perform only a service-level access control and therefore abandon the reservation per session mode. The IMS-based $\mathrm{SP}$ verifies if the VoIP service platform can accommodate the call, based only on information such as the number of active sessions and the number of supported simultaneous sessions (especially within nodes embedding border gateway function (BGF), such as session border controllers (SBCs).

Figure 6a illustrates an IMS-based telephony service provider in which BGF nodes are interconnected through the infrastructure of an INP supporting several network planes. Connectivity provisioning aspects are negotiated between the IMS-based telephony SP and the underlying NP including code points (e.g., DSCP) for marking the SP generated traffic. To honor the IMS SP connectivity requirements (including QoS and robustness), the underlying INP engineers an NP suitable for transport of conversational services traffic. The creation of the NP is opaque to the IMS SP. We provide examples of engineering NPs.

To benefit from these connectivity guarantees, the BGF nodes must mark the outbound traffic with the DSCP code(s) as agreed during the CPA phase. When this traffic enters the INP domain, it is classified accordingly and is bound to the conversational services NP. This traffic is then delivered to the next BGF node and guarantees are met due to the NP technical realizations means.

To offer QoS-enabled conversational services world-wide, IMS-based SPs must interconnect together but also require that the underlying IP infrastructure be engineered in an appropriate manner. Due to the deployment of parallel Internets, a coherent end-to-end QoS treatment is provided across several INPs. Concretely and for illustration purposes, IMS SP1 and IMS SP2 should agree CPAs with their respective underlying INPs. QoS, robustness, and the scope of the guarantees are part of these CPAs. These CPAs are implemented by engineering corresponding NPs and their binding with external ones to build a PI as illustrated in Fig. 6b. Owing to the deployment of this PI, conversational traffic will benefit from an inter-domain QoS treatment. Note that two scenarios for building this PI are provided in a previous section. The first scenario is suitable for strict QoS guarantees, and every intermediate domain must have a VoIP-friendly NP. The second scenario provides enhanced QoS but without guarantees (intermediate domains are not assumed to implement VoIPfriendly NPs), and best effort treatment is sufficient.

\section{CONCLUSIONS}

This article has proposed an approach to ease the introduction of differentiated services not only by performing at the forwarding level but also by tuning multi-dimensional techniques at the routing and resource management levels. The concepts of network plane and parallel Internets are introduced. We presented a busi-
${ }^{4}$ TS23.207 uses the notion of "IP bearer services" but never defines it. This notion largely predates the $3 G P P$ work. In I210 ISDN Recommendation (1993), bearer services are introduced as opposed to teleservices. A bearer service is a network point-to-point relationship. In IP networks, this notion has no real meaning; any host is ready to communicate with any other host in the world, and when a communication is established between two hosts, it normally brings no particular states in the network (according to the so-called fate sharing property). That would mean a host always has bearer services with all other stations in the Internet. 


Two scenarios to
build parallel
Internets are
provided. The first
scenario is based on
the use of PDB,
meta-QoS-class, and
QoS-enhanced BGP.
The second scenario
employs a
combination of
multi-topology
routing and IP
tunneling
techniques.

ness model that captures the business actors and their relationship. The adopted business model assumes a decoupling between the service provider and IP network provider roles. In addition, the AGAVE functional architecture is described, including the functions required to offer differentiated services. Functions for engineering network planes and parallel Internets in order to satisfy heterogeneous QoS requirements set by SPs are presented in detail. Two scenarios to build parallel Internets are provided. The first scenario is based on the use of PDB, meta-QoS-class, and QoS-enhanced BGP. The second scenario employs a combination of multi-topology routing and IP tunneling techniques. This article also identified QoS problems in 3GPP architecture and proposed a framework to ease the implementation of QoS-enabled multimedia services.

The merits of the presented approach are as follows. First, it advocates decoupling service functions from control functions by specifying simplified interfaces between the two. Second, it is lightweight for the SP because the complexity is pushed to the INP. Third, the approach is deterministic due to the presence of the NP emulation function, which assesses the status of the network and evaluates the impact of introducing new NPs and accepting new IP-connectivity provisioning requests. Fourth, it abolishes the node-centric provisioning/configuration approach in favor of network-based configuration, because the NP provisioning and maintenance generates the whole NP configuration, ensuring configuration consistency. Fifth, INP easily can evaluate the interference between service activation requests based on the analysis of service requirements.

\section{ACKNOWLEDGMENTS}

This work was undertaken in the Information Society Technologies (IST) AGAVE project, which is partially funded by the European Commission. We would like to thank all our AGAVE colleagues who contributed to the ideas presented here.

\section{REFERENCES}

[1] N. Chiappa, "IPng Technical Requirements of the Nimrod Routing and Addressing Architecture," RFC 1753, Dec. 1994.

[2] B. Braden, D. Clark, and S. Shenker, "Integrated Services in the Internet Architecture: An Overview," RFC 1633, June 1994.

[3] S. Blake et al., "An Architecture for Differentiated Services," RFC 2475, Dec. 1998.

[4] G. Camarillo and M. A. Garcia-Martin, The 3G IP Multimedia Subsystem - Merging the Internet and the Cellular Worlds, Wiley, 2005.

[5] TISPAN, "Telecommunications and Internet converged Services and Protocols for Advanced Networking, NGN Release 1," TR180001, 2006.

[6] G. Camarillo et al., "Integration of Resource Management and Session Initiation Protocol (SIP)," RFC 3312, Oct. 2002.

[7] M. Boucadair et al., "Parallel Internets Framework," AGAVE deliv. D1.1, Sept. 2006.

[8] E. Mykoniati et al., "Initial Specification of the Connectivity Service Provisioning Interface Components," AGAVE deliv. D2.1, Nov. 2006.

[9] P. Psenak et al., "MT-OSPF: Multi Topology (MT) Routing in OSPF," Internet draft, draft-ietf-ospf-mt, work in progress.

[10] T. Bates et al., "Multiprotocol Extensions for BGP-4," RFC 2858, June 2000.
[11] Callejo-Rodríguez et al., "A Decentralized Traffic Management Approach for Ambient Networks Environments," 16th IFIP/IEEE Int'l. Wksp. Distrib. Sys.: Ops. and Mgmt., 2005.

[12] M. Boucadair, "QoS-Enhanced Border Gateway Protocol," Internet draft, draft-boucadair-qos-bgp-spec, work in progress.

[13] B. Quoitin and O. Bonaventure, "A Cooperative Approach to Interdomain Traffic Engineering," Proc. NGI'05.

[14] A. Raj and O. C. Ibe, "A Survey of IP and Multiprotocol label Switching Fast Reroute Schemes," to appear, Journal of Computer Networks.

[15] P. Levis et al., "A New Perspective for a Global QoS-Based Internet," J. Commun. Software and Sys., Nov. 2005.

\section{BIOGRAPHIES}

MOHAMED BOUCADAIR (mohamed.boucadair@orangeftgroup.com) graduated from the École Nationale Supérieure d'Ingénieur de Caen (Institut des Sciences de la Matière et des Rayonnements), a French school for engineers. He is an R\&D engineer with France Telecom R\&D and formerly worked on dynamic provisioning, QoS, multicast, and intra/interdomain traffic engineering. He now works on VolP services.

PIERRE LEVIS (Pierre.levis@orange-ftgroup.com) has been with France Telecom, R\&D Division since 1998. He has worked in the specification, development, and evaluation of IP service offerings. His research interests are QoS, IPv6, network security, AAA, and mobile networks. He was an assistant professor atInstitut National des Telecommunications, Evry France, from 1990 to 1998, where he was in charge of computer network courses, and his research interest was information technology.

DAVID GRIFFIN (dgriffin@ee.ucl.ac.uk) has a B.Sc. in electrical engineering from Loughborough University, United Kingdom, and currently is completing a Ph.D. on a part-time basis in electrical engineering from the University of London. $\mathrm{He}$ is a senior research fellow in the Department of Electronic and Electrical Engineering, University College London (UCL). Before joining UCL he was a systems design engineer at GEC-Plessey Telecommunications, United Kingdom, and a researcher at the Foundation for Research and Technology - Hellas, Institute of Computer Science, Crete, Greece.

NING WANG (n.wang@surrey.ac.uk) holds a B.Eng. in computer science from Changchun University of Science and Technology, P.R. China, an M.Eng. in electrical and computer engineering from Nanyang Technological University, Singapore, and a Ph.D. in electronic engineering from the University of Surrey. $\mathrm{He}$ is a research fellow at the Centre for Communication Systems Research (CCSR), University of Surrey.

MichaEl HowARTH (m.howarth@surrey.ac.uk) holds a Bachelor's degree in engineering science and a D.Phil in electrical engineering, both from Oxford University, and an M.Sc. in telecommunications from the University of Surrey. He is a lecturer in networking at the Centre for CCSR, University of Surrey.

GeORGe PAVlou (g.pavlou@surrey.ac.uk) holds a Diploma in engineering from the National Technical University of Athens, Greece, and M.Sc. and Ph.D. degrees from University College London. He is a professor of communication and Information Systems at CCSR, University of Surrey, where he leads the activities of the Networks Research Group. His research interests focus on network management, networking, and service engineering.

ELENI MYKonIATI (eleni_mykoniati@egreta.gr) received a B.Sc. in computer science from Piraeus University in 1996 and a Ph.D. degree with specialization in admission control for QoS in IP networks from National Technical University of Athens in 2003. Since 2004 she has worked for Algonet S.A. She worked as a research associate for Telscom S.A. Switzerland, and the DB and Telecom Laboratories of NTUA until 2003. She has been involved in REFORM, EXPERTVIKING, TEQUILA, MESCAL, and AGAVE; working on signaling, network element adaptation, admission control, SLA modeling, and business-driven traffic engineering.

PANOS GEORGATSOS (pgeorgat@egreta.gr) received a B.SC. degree in mathematics from the National University of Athens and a Ph.D. in computer science, with specializa- 
tion in data network routing and performance analysis through queuing theory techniques, from Bradford University, United Kingdom. He leads the Telecommunications R\&D Group within Algonet SA, Greece. He has acted as a consultant for the Hellenic Telecommunications Organization (OTE) in several projects.

BRUNO QUOITIN (bruno.quoitin@uclouvain.be) received his B.S. degree in mathematics and his M.S. degree in computer science from Facultes Universitaires Notre-Dame de la Paix in Namur in 1999. He obtained his Ph.D. from Université Catholique de Louvain, Belgium, in 2006. He is a research fellow at Université Catholique de Louvain. His current research is in the area of interdomain routing and network modeling. He is the author of the C-BGP simulator.

JORGE RODRIGUEZ SANCHEZ (jrs327@tid.es) received his Master's degree in telecommunication engineering from the Universidad Carlos III de Madrid, Spain, in 2006. Since
2005 he has been working for Telefónica I+D in Advanced Network Planning, working on traffic engineering and traffic control for IP networks, paying particular attention to the problem of quality of service differentiation and routing algorithms. He was awarded the Best Final Project in Information Technologies and Communications Applied to Banking by the Spanish Telecommunication Engineer Association for his final project.

MARIA LUISA GARCIA OSMA (mlgo@tid.es) received her Master's degree in telecommunications engineering from the Universidad Politécnica de Madrid in 2000. She is currently working for Telefónica S. A. in the strategy, innovation, and business development area. From 1999 to 2007 she worked for Telefónica I+D in Network and Services Planning, studying diverse topics, including the study, evaluation, and design of traffic engineering technologies for IP networks, including routing mechanisms and protocols, IPV4/IPv6 transition, dimensioning methods for UMTS IP networks, and so on. 